www.mjn.mosuljournals.com

\title{
Assessment of nurses knowledge and practices concerning cardiogenic shock
}

\section{Article information}

\section{Article history:}

Received March 13, 2018

Accepted August 1, 2019

Available online March 17, 2018

\author{
DOI: 10.33899/mjn.2018.162887 @2020, College of Nursing, University of Mosul. \\ Creative Commons Attribution 4.0 International License \\ https://mjn.mosuljournals.com/article_168249.html
}

Jasim Mohamed Taib AL-Hayali ${ }^{1}$

Ahmed Abdulla Aziz -AL-samady ${ }^{2}$

\begin{abstract}
The nurses have an important and effective role in the evaluation of the type of nursing care, which are providing to patient with cardiogenic shock, via their contribution in the quality of nursing care, which are provided to cardiogenic shock patients. The present study aims at assessment of knowledge and practices of nurses that they working in the coronary care unit and emergency department.

A Correlation study was performed in the coronary care unit and emergency department in Ibn Sena teaching hospital , Almousl general hospital and aljmhori teaching hospital in mousl city for the period from 8 january 2005 to 12 may 2005. The sample of the study included (50 subject) of nurses who work in he above units. The results of the study showed that there was significant difference between the knowledge and practice of nurses in regard to theoretical mean.. The level of education . and duration of employment had appositive impact on the knowledge and practices. While the gender and the training session had no significant impact on knowledge and practice.

Based on the above results, the study recommends that the nurses in these units should haven sufficient practice and the knowledge update about critical care, especially about cardiogenic shock, nursing staff who work in the coronary care unit and emergency department units should be assessing after tanning; and larger study to elaborate the effects of different variables on the knowledge and practice of nurses.
\end{abstract}

\footnotetext{
${ }^{1}$ Assistant Professor

${ }^{2}$ The Higher Institute of Health in Mosul / Nineveh Health Department
} 


\section{Mosul Journal of Nursing, Vol. 6, No. 2, 2018 ( 100-111 )}

\section{Introduction}

Cardiogenic shock is the Armageddon of heart disease. Most of these patients die with in 24 hours, few live with in 24 host of these patients die with in 24 hours, few live with in 24 days.

Myocardial infarction (MI) is the most common cause of cardiogenic shock in modern times. Clinicians recognized cardiogenic shock as a laow cardiac out put state secondary to extensive left ventricular infarction, development of a mechanical defect (sharma Sat,etal, 2004). Cardiogenic shock is typically the result of a massive of damage to the left ventricular myocardium.(Holmes, 2003). The systemic nursing process is an essential perquisite for providing effective care for patients in shock( Lancaster, 1990)

Critical care nursing is the specialty within nursing that deals specifically with human responses to life $\quad-$ threatening problems .( Hartshorn and Lambor, 1993). The adult critical care nurse is responsible for managing the care of adult and elderly patients experiencing life - threating problems requiring complex assessment, high intensity therapies and interventions, and continuous nursing vigilance. Nursing staff in the critical care nursing are enhancing by an atmosphere of teamwork, and it will improve standers of care Patients hospitalized with acute myocardial infarction , 7-10\% (AMI) develop cardiogenic shock.(Hochman, etal, 1999). The precise incidence is difficult to measure because some patients die before reaching the hospital . Inconstant, early and intensive monitoring can increase the apparent incidence of cardiogenic shock of $7.5 \%$; this incidence remained stable from 1975 to 1988. In the Global utilization of streptokinase (GUSTO-1) trial, the incidence of cardiogenic shock was $7.2 \%$, a rate similar to that found in other multicenter thrombolytic trials.(Hollenberg,et al, 1999). Shock develops in most patients of AMI within the next 48 hour. In multicenter, country - wide survey from Denmark , 59 ide $\%$ of patients developed shock within 48 hours, but $30 \%$ developed shock more than 4 days after MI . As might be expected, prior MI ; older age ; diabetes mellitus ; female gender ; and a history of angina pectoris ; stroke ; or peripheral vascular disease have been associated with increased incidence of cardiogenic shock, but the predictive power of these factors in an individual patient is Limited.(Goldman,2004).

\section{Rational}

Cardiogenic shock is a critical state requires efficient care. Nurse plays an important role in the management of cardiogenic shock, the exact role of the nurse during the management of patients with cardiogenic shock is not clear, that is why the outcomes of intervention was not up the standard expected. The nurse at the bed side must have the knowledge and skill to evaluate the total picture of patients status if she is to fulfill the role of alerting physician to significant changes in the patients condition.

Objectives of the study : 


\section{Mosul Journal of Nursing, Vol. 6, No. 2, 2018 ( 100-111 )}

To assess the nurses Knowledge and practices during the work in coronary care unit (CCU) and emergency department (ED) in Mousl city.

To determine the relationship between the characteristics of the nurse as ( age, the gender, place of work, training sessions, level of education, duration of employment , number of employers, time of work) in coronary care unit and emergency department with their Knowledge and practices.

\section{Methodology:}

\section{Administrative Arrangements:}

Prior to the actual collection of data, a formal administrative approval was obtained to conduct the study from the ministry of health , department of health in Nineveh health office in order to conduct the study in Ibn Sena teaching hospital, Almousl general hospital and aljmhori teaching hospital under number(15683).

\section{Setting of the study:}

The study was carried out in Ibn Sena teaching hospital- coronary care unit| (CCU)- Almousl general hospital (CCU\&ED), and aljmhori teaching hospital (ED).

\section{Design of the study:}

Correlation descriptive study

\section{Sample of the study :}

The sample of the study consists of (50) nurses choose purposively who are working in ibn sena teaching hospital (CCU) (16 nurse), aljmhori teaching hospital (ED) ( 15 nurse)and almousl general hospital CCU and ED (19 nurse).

\section{Data collection :}

The data, which were collected in the (3) hospitals for the period from 15 February to 12 may 2005 include in sequence :

*Socio - demographic characteristic, which include 8 variables : age, the gender, place of work, duration of employment, training session, level of education, number of employers, time of work.

*The questionnaire about cardiogenic shock was presented to the nurses to fill in during the period from 15 February to 2 march

*the practice assessment done by the investigator through indirect observation during performance of the tasks, assessment of practices was carried out began at 15 march to 12 may 2005.

\section{Tool of the study:}

Special questionnaire was prepared by the investigator according to the expert opinion, literature review about cardiogenic shock from the books, internet, and preliminary study which was carried out in Ibn Sena teahing hospital, Almousl general hospital and aljmhori teaching hospital
A- Knowledge assessment questionnaire ( closed ended questions) consists of:
1- General concepts of cardiogenic $\operatorname{shock}(17$ sub items)
2- Information about care of cardiogenic shock (20 sub items)
3- Knowledge about medications (8 sub items)
4- General nursing information $(8$ sub items)

\section{B- Practice assessment}




\section{Mosul Journal of Nursing, Vol. 6, No. 2, 2018 ( 100-111 )}

The tasks of blood pressure, oxygen administration . and cannula insertion are example for assessing the practice of the study, because these tasks are standard in the management of cardiogenic shock, three checklists are lists are constructed by ( clinical nursing skills and technique, 2002) were used for the purpose of evaluation and statistical measurement.

Testing the validity and reliability of the tool:

\section{Content validity :}

Prior collecting the data of the study, the tools were examined to identify their validity . the investigator submitted the tools

\section{Results}

Table (4-1) demographic data of the study sample

\begin{tabular}{|c|c|c|c|c|}
\hline Characteristics & Items & No. & $\%$ & Mean \pm SD \\
\hline \multirow{4}{*}{$\begin{array}{l}\text { Age groups } \\
\text { (years) }\end{array}$} & $21-30$ & 23 & 46.0 & $26.22 \pm 1.37$ \\
\hline & $31-40$ & 14 & 28.0 & $35.79 \pm 2.67$ \\
\hline & 40 & 13 & 26.0 & $45.31 \pm 2.90$ \\
\hline & & Total & & $33.86 \pm 8.28$ \\
\hline \multirow[t]{2}{*}{$\operatorname{sex}$} & Male & 44 & 88.0 & \\
\hline & female & 6 & 12.0 & \\
\hline \multirow[t]{2}{*}{ place } & $\mathrm{CCU}$ & 26 & 52.0 & \\
\hline & Emergency & 24 & 48.0 & \\
\hline Duration & 5 & 35 & 70.0 & \\
\hline \multirow{2}{*}{$\begin{array}{l}\text { employment } \\
\text { (year) }\end{array}$} & $6-10$ & 7 & 14.0 & \\
\hline & 10 & 8 & 16.0 & \\
\hline \multirow[t]{3}{*}{ Training session } & no & 18 & 36.0 & \\
\hline & yes & 32 & 64.0 & \\
\hline & Non degree trainin & 4 & 8.0 & \\
\hline \multirow[t]{5}{*}{ Level of education } & course & & & \\
\hline & School of nursing & 27 & 54.0 & \\
\hline & Nursing institute & 10 & 20.0 & \\
\hline & Nursing college & 9 & 18.0 & \\
\hline & 3 & 12 & 24.0 & \\
\hline \multirow[t]{3}{*}{ No.of employers } & 6 & 8 & 16.0 & \\
\hline & 7 & 30 & 60.0 & \\
\hline & Day & 37 & 74.0 & \\
\hline \multirow[t]{2}{*}{ Time } & Evening & 8 & 16.0 & \\
\hline & night & 5 & 10.0 & \\
\hline
\end{tabular}

This table show that most of the nurses (46.0\%) are between the ages (21-30) years. to a panel of (12) experts in different fields of knowledge.

\section{Reliability (pilot study):}

The pilot study was carried out on (6) nurse which were selected from the schedule of nurses working in the CCU, ED (in Ibn Sena teahing hospital , Almousl general hospital and aljmhori teaching hospital) Evaluation of responses and statistical analysis between the test and retest on the same sample of nurses done by pearson,s coefficient for items in the studied group which showed that reliability estimate for knowledge $(0.967)$ and for practice was $(0.821)$ between test and retest. 


\section{Mosul Journal of Nursing, Vol. 6, No. 2, 2018 ( 100-111 )}

nurse were working in the CCU and $48 \%$ in the ED. Regarding duration of employment , those with less than (5 years) constituted $70 \%$, (6-10\%) 14\%, and above 10 years $16 \%$. Concerning the level of education high percentage of nurses with nursing institute $54 \%$ were graduate of school, while $20 \%$ were nurses with nursing institute, the nurse from nursing college were (9) which represent (18\%), and the remaining nurses represent $(8 \%)$. Concerning the number of employers 3 nurses represent $(24 \%), 6$ nurses which represented $16 \%, 7$ nurse which represented $60 \%$. Regarding the time of work the day constituted $74 \%$, evening constituted $16 \%$, night constituted $10 \%$.

Table (4-2) comparison between the mean of knowledge about cardiogenic shock with the theoretical mean by using test.।

\begin{tabular}{|c|c|c|c|c|}
\hline knowledge & Mean \pm SD & $\begin{array}{l}\text { theoretical } \\
\text { mean }\end{array}$ & t- value & $\mathrm{p}$ - value \\
\hline $\begin{array}{ll}\text { General information } & \text { about } \\
\text { cardiogenic shock } & \end{array}$ & $9.18 \pm 2.93$ & 8.5 & 1.64 & $\begin{array}{r}>0.001 \\
(\mathrm{NS})\end{array}$ \\
\hline $\begin{array}{l}\text { Information about care of } \\
\text { cardiogenic shock }\end{array}$ & $12.22 \pm 3.48$ & 10.0 & 4.51 & $<0.001$ \\
\hline Information about medications & $5.14 \pm 1.58$ & 4.0 & 4.0 & $<0.001$ \\
\hline General nursing information & $5.38 \pm 1.32$ & 4.0 & 4.0 & $<0.001$ \\
\hline
\end{tabular}

This table show a significant difference at $\mathrm{p}$ value $<0.001$ by using $\mathrm{t}-$ test between mean of knowledge of nurses about cardiogenic shock with regard to theoretical means .

Table (4-3) comparison between the mean of practice with theoretical mean by using $t$ test

\begin{tabular}{|c|c|c|c|c|}
\hline practice & Mean \pm SD & $\begin{array}{l}\text { theoretical } \\
\text { mean }\end{array}$ & t- value & $\mathrm{p}$ - value \\
\hline Blood pressure measurement & $9.94 \pm 2.00$ & 7.5 & 8.61 & $<0.001$ \\
\hline Oxygen administration & $6.42 \pm 1.09$ & 4.5 & 12.46 & $<0.001$ \\
\hline Cannula insertion & $5.72 \pm 0.70$ & 3.5 & 22.39 & $<0.001$ \\
\hline
\end{tabular}

This table show a significant difference at $\mathrm{p}$ value $<0.001$ by using $\mathrm{t}-$ test between mean of practice of nurses about cardiogenic shock with regard to theoretical means .

Table (4-4) comparison of work performance during management of cardiogenic shock and ordinary time

\begin{tabular}{l|llll}
\hline \multicolumn{1}{c|}{ practices } & \multicolumn{2}{|c}{ Mean \pm SD } & t- calculated & Level of sig. \\
\cline { 2 - 3 } & Ordinary time & Shock time & & \\
\cline { 1 - 2 } $\begin{array}{l}\text { Blood pressur } \\
\text { measurement }\end{array}$ & $64.44 \pm 5.44$ & $76.00 \pm 10.04$ & 2.58 & $<0.05$ \\
$\begin{array}{l}\text { Oxygen } \\
\text { administration }\end{array}$ & $70.37 \pm 9.07$ & $71.11 \pm 11.94$ & 0.13 & $>0.05$ (NS) \\
$\begin{array}{l}\text { Cannula insertion } \\
\text { Cann }\end{array}$ & $80.95 \pm 14.75$ & $82.68 \pm 6.02$ & 0.37 & $>0.05(\mathrm{NS})$
\end{tabular}

Table (4-5) comparison of nurses knowledge according to their age by using ANOVA -test

\begin{tabular}{l|lcl}
\hline Age/ knowledge & $\begin{array}{l}\text { Age } \\
\text { (year) }\end{array}$ & Mean \pm SD & F-calculatec Level of sig. \\
\hline & $21-30$ & $51.66 \pm 14.83$ &
\end{tabular}




\section{Mosul Journal of Nursing, Vol. 6, No. 2, 2018 ( 100-111 )}

\begin{tabular}{|c|c|c|c|c|}
\hline $\begin{array}{l}\text { General information about cardiogeni } \\
\text { shock }\end{array}$ & $\begin{array}{l}31-40 \\
>40\end{array}$ & $\begin{array}{l}55.46 \pm 17.98 \\
56.56 \pm 21.01\end{array}$ & 0.40 & $>0.05(\mathrm{NS})$ \\
\hline $\begin{array}{l}\text { Information about care of cardiogeni } \\
\text { shock }\end{array}$ & $\begin{array}{l}21-30 \\
31-40 \\
>40\end{array}$ & $\begin{array}{l}61.30 \pm 20.29 \\
61.43 \pm 15.25 \\
60.38 \pm 15.06\end{array}$ & 0.01 & $>0.05(\mathrm{NS})$ \\
\hline Information about medications & $\begin{array}{l}21-30 \\
31-40 \\
>40\end{array}$ & $\begin{array}{l}61.96 \pm 21.81 \\
66.07 \pm 15.83 \\
66.35 \pm 20.66\end{array}$ & 0.28 & $>0.05(\mathrm{NS})$ \\
\hline General nursing information & $\begin{array}{l}21-30 \\
31-40 \\
>40\end{array}$ & $\begin{array}{l}69.57 \pm 17.20 \\
64.29 \pm 17.58 \\
66.35 \pm 14.78\end{array}$ & 0.46 & $>0.05(\mathrm{NS})$ \\
\hline
\end{tabular}

Table (4-6) comparison of nurses practices according to their age by using ANOVA -test

\begin{tabular}{l|llll}
\hline Age/ practices & $\begin{array}{l}\text { Age } \\
\text { (year })\end{array}$ & Mean \pm SD & F-calculatec Level of sig. \\
\hline Blood pressure measurement & $21-30$ & $64.64 \pm 16.87$ & & \\
& $31-40$ & $68.10 \pm 7.92$ & 0.32 & $>0.05$ (NS) \\
& $>40$ & $67.18 \pm 11.37$ & & \\
Oxygen administration & $21-30$ & $68.12 \pm 13.94$ & 1.73 & $>0.05$ (NS) \\
& $31-40$ & $75.40 \pm 9.92$ & & \\
Cannula insertion & $>40$ & $72.65 \pm 9.75$ & & \\
& $21-30$ & $77.46 \pm 9.46$ & 4.39 & $<0.05$ \\
& $31-40$ & $83.67 \pm 11.00$ & & \\
& $>40$ & $68.81 \pm 7.05$ & &
\end{tabular}

Table (4-7) comparison of nurses knowledge according to their gender by using $t$-test

\begin{tabular}{|c|c|c|c|c|}
\hline \multirow[t]{2}{*}{ gender/ knowledge } & \multicolumn{2}{|c|}{ Mean \pm SD } & & Level \\
\hline & male & female & calculated & sig. \\
\hline $\begin{array}{l}\text { General information about cardiogeni } \\
\text { shock }\end{array}$ & $53.88 \pm 15.84$ & $54.90 \pm 27.51$ & 0.14 & $>0.05(\mathrm{NS})$ \\
\hline $\begin{array}{l}\text { Information about care of cardiogeni } \\
\text { shock }\end{array}$ & $61.59 \pm 17.18$ & $57.50 \pm 20.19$ & 0.54 & $>0.05(\mathrm{NS})$ \\
\hline Information about medications & $46.49 \pm 19.98$ & $62.50 \pm 19.37$ & 0.23 & $>0.05(\mathrm{NS})$ \\
\hline General nursing information & $67.90 \pm 16.04$ & $62.50 \pm 20.92$ & 0.75 & $>0.05(\mathrm{NS})$ \\
\hline
\end{tabular}

Table (4-8) comparison of nurses practices according to their gender by using $t$-test

\begin{tabular}{l|lllll}
\hline gender/ practices & \multicolumn{3}{c}{$\begin{array}{c}\text { Mean } \pm \text { SD } \\
\text { female }\end{array}$} & $\begin{array}{l}\text { t- } \\
\text { calculated }\end{array}$ & $\begin{array}{l}\text { Level } \\
\text { sig. }\end{array}$ \\
\hline Blood pressure measurement & $66.67 \pm 11.94$ & $63.33 \pm 22.61$ & 0.57 & $>0.05(\mathrm{NS})$
\end{tabular}


Oxygen administration

$\begin{array}{lll}70.96 \pm 11.79 & 74.07 \pm 15.187 & 0.59\end{array}$

$>0.05$ (NS)

Cannula insertion

$81.17 \pm 10.11 \quad 85.71 \pm 9.04$

1.04

$>0.05(\mathrm{NS})$

Table (4-9) comparison of nurses knowledge according to their place of work by using $t$ -test

\begin{tabular}{|c|c|c|c|c|}
\hline \multirow[t]{2}{*}{ Place of work/ knowledge } & \multicolumn{2}{|c|}{ Mean +SD } & & Level \\
\hline & CCU $n=26$ & $E D n=24$ & calculated & \\
\hline $\begin{array}{l}\text { General information about cardiogeni } \\
\text { shock }\end{array}$ & $58.14 \pm 17.87$ & $49.51 \pm 15.70$ & 1.81 & $>0.05(\mathrm{NS})$ \\
\hline $\begin{array}{l}\text { Information about care of cardiogeni } \\
\text { shock }\end{array}$ & $61.73 \pm 18.05$ & $60.42 \pm 17.00$ & 0.26 & $>0.05(\mathrm{NS})$ \\
\hline Information about medications & $68.27 \pm 17.76$ & $59.90 \pm 21.17$ & 1.52 & $>0.05(\mathrm{NS})$ \\
\hline General nursing information & $73.08 \pm 15.29$ & $60.94 \pm 15.77$ & 2.76 & $<0.05$ \\
\hline
\end{tabular}

Table (4-10) comparison of nurses knowledge according to their place of work by using t -test

\begin{tabular}{|c|c|c|c|c|}
\hline \multirow[t]{2}{*}{ Place of work/ practices } & \multicolumn{2}{|c|}{ Mean +SD } & t- calculated & Level \\
\hline & CCU n=26 & $\mathrm{ED} n=24$ & & \\
\hline Blood pressure measurement & $69.74+10.02$ & $62.50+15.58$ & 1.97 & $>0.05(\mathrm{NS})$ \\
\hline Oxygen administration & $72.65+11.43$ & $69.91+12.89$ & 0.80 & $>0.05(\mathrm{NS})$ \\
\hline Cannula insertion & $82.97+9.91$ & $80.36+10.16$ & 0.92 & $>0.05(\mathrm{NS})$ \\
\hline
\end{tabular}

Table (4-11) comparison of nurses knowledge according to their duration of employment by using ANOVA -test

\begin{tabular}{|c|c|c|c|c|}
\hline $\begin{array}{l}\text { duration of employment } \\
\text { knowledge }\end{array}$ & duration(year) & Mean \pm SD & $\begin{array}{l}\text { F- } \\
\text { calculated }\end{array}$ & Level of sig. \\
\hline $\begin{array}{l}\text { General information abou } \\
\text { cardiogenic shock }\end{array}$ & $\begin{array}{c}<5 \\
6-10 \\
>10\end{array}$ & $\begin{array}{l}51.43 \pm 15.12 \\
57.98 \pm 20.21 \\
61.76 \pm 22.45\end{array}$ & 1.41 & $>0.05$ (NS) \\
\hline $\begin{array}{l}\text { Information about care } \\
\text { cardiogenic shock }\end{array}$ & $\begin{array}{c}<5 \\
6-10 \\
>10\end{array}$ & $\begin{array}{l}56.86 \pm 17.83 \\
75.71 \pm 38 \\
66.88+13.08\end{array}$ & 4.53 & $<0.05$ \\
\hline Information about medications & $\begin{array}{c}<5 \\
6-10 \\
>10\end{array}$ & $\begin{array}{l}60.36 \pm 18.56 \\
82.14 \pm 17.47 \\
65.63 \pm 19.76\end{array}$ & 4.02 & $<0.05$ \\
\hline General nursing information & $\begin{array}{c}<5 \\
6-10 \\
>10\end{array}$ & $\begin{array}{l}67.86 \pm 17.23 \\
64.29 \pm 16.81 \\
67.19 \pm 14.85\end{array}$ & 0.13 & $>0.05(\mathrm{NS})$ \\
\hline
\end{tabular}


Table (4-12) comparison of nurses practices according to their duration of employment by using ANOVA -test

\begin{tabular}{l|cccc}
\hline duration of employment/ practices & duration(year) & Mean \pm SD & $\begin{array}{l}\text { F- } \\
\text { calculated }\end{array}$ & Level of sig. \\
\hline Blood pressure measurement & $<5$ & $63.24 \pm 13.56$ & 3.96 & \\
& $6-10$ & $77.14 \pm 7.56$ & & $<0.05$ \\
Oxygen administration & $>10$ & $70.00 \pm 11.27$ & & \\
& $<5$ & $69.52 \pm 12.15$ & 1.34 & $>0.05$ (NS) \\
& $6-10$ & $76.19 \pm 13.50$ & & \\
Cannula insertion & $>10$ & $75.00 \pm 9.85$ & & \\
& $<5$ & $79.59 \pm 10.56$ & 2.89 & $>0.05$ (NS) \\
& $6-10$ & $85.71 \pm 8.25$ & & \\
& $>10$ & $87.50 \pm 5.05$ & &
\end{tabular}

Table (4-13) comparison of nurses knowledge according to their training sessions of work by using $t$-test

\begin{tabular}{|c|c|c|c|c|}
\hline \multirow[t]{2}{*}{ Place of work/ knowledge } & \multicolumn{2}{|c|}{ Mean \pm SD } & \multirow{2}{*}{$\begin{array}{l}\mathrm{t}- \\
\text { calculated }\end{array}$} & \multirow{2}{*}{$\begin{array}{ll}\text { Level } & 0 \\
\text { sig. } & \end{array}$} \\
\hline & No $n=18$ & yes $n=32$ & & \\
\hline $\begin{array}{l}\text { General information abou } \\
\text { cardiogenic shock }\end{array}$ & $50.00 \pm 16.05$ & $56.25 \pm 17.74$ & 1.24 & $>0.05(\mathrm{NS})$ \\
\hline $\begin{array}{l}\text { Information about care of cardiogeni } \\
\text { shock }\end{array}$ & $58.89 \pm 17.11$ & $62.34 \pm 17.69$ & 0.67 & $>0.05(\mathrm{NS})$ \\
\hline Information about medications & $63.19 \pm 20.32$ & $64.84 \pm 19.68$ & 0.28 & $>0.05(\mathrm{NS})$ \\
\hline General nursing information & $65.97 \pm 16.50$ & 67.97. \pm 19.78 & 0.41 & $>0.05(\mathrm{NS})$ \\
\hline
\end{tabular}

Table (4-12) comparison of nurses practices according to their training sessions of work by using $\mathrm{t}$-test

\begin{tabular}{|c|c|c|c|c|}
\hline \multirow[t]{2}{*}{ Place of work/ practices } & \multicolumn{2}{|c|}{ Mean \pm SD } & t- calculated & Level \\
\hline & No $n=18$ & yes $n=32$ & & \\
\hline Blood pressure measurement & $65.93 \pm 15.15$ & $66.46 \pm 12.50$ & 0.13 & $>0.05(\mathrm{NS})$ \\
\hline Oxyg & $70.99 \pm 12.72$ & $71.53 \pm 11.95$ & 0.15 & $>0.05(\mathrm{NS})$ \\
\hline Cannula insertion & $79.73 \pm 11.20$ & $83.04 \pm 6.21$ & 1.25 & $>0.05(\mathrm{NS})$ \\
\hline
\end{tabular}

Table (4-14) comparison of nurses knowledge according to their level of education by using ANOVA -test

\begin{tabular}{l|lrll}
\hline level of education / knowledge & level of education & Mean \pm SD & $\begin{array}{l}\text { F- } \\
\text { calculated }\end{array}$ & $\begin{array}{l}\text { Level o } \\
\text { sig. }\end{array}$ \\
\hline $\begin{array}{l}\text { General information abou } \\
\text { cardiogenic shock }\end{array}$ & $\begin{array}{l}\text { Non degree trainin } \\
\text { course }\end{array}$ & & & \\
School of nursing & $52.65 \pm 15.47$ & 1.14 & $>0.05$ \\
& & & (NS)
\end{tabular}




\begin{tabular}{|c|c|c|c|c|}
\hline \multirow{4}{*}{$\begin{array}{l}\text { Information about care } \\
\text { cardiogenic shock }\end{array}$} & Nursing institute & $57.06 \pm 18.82$ & \multirow{5}{*}{2.42} & \multirow{5}{*}{$\begin{array}{l}>0.05 \\
(\mathrm{NS})\end{array}$} \\
\hline & Nursing college & $60.13 \pm 16.58$ & & \\
\hline & $\begin{array}{l}\text { Non degree trainin } \\
\text { course }\end{array}$ & $45.00 \pm 70.07$ & & \\
\hline & $\begin{array}{l}\text { School of nursing } \\
\text { Nursing institute }\end{array}$ & $\begin{array}{l}58.89 \pm 16.37 \\
69.00 \pm 16.96\end{array}$ & & \\
\hline \multirow{3}{*}{ Information about medications } & Nursing college & $66.11 \pm 16.65$ & & \\
\hline & $\begin{array}{l}\text { Non degree trainin } \\
\text { course }\end{array}$ & $43.75 \pm 21.65$ & \multirow[t]{3}{*}{2.51} & $\begin{array}{l}>0.05 \\
(\mathrm{NS})\end{array}$ \\
\hline & $\begin{array}{l}\text { School of nursing } \\
\text { Nursing institute }\end{array}$ & $\begin{array}{l}26.96 \pm 16.80 \\
73.75 \pm 18.11\end{array}$ & & \\
\hline \multirow{5}{*}{ General nursing information } & Nursing college & $66.67 \pm 24.21$ & & \\
\hline & $\begin{array}{l}\text { Non degree trainin } \\
\text { course }\end{array}$ & $43.75 \pm 12.50$ & \multirow[t]{4}{*}{5.03} & $<0.05$ \\
\hline & School of nursing & $66.20 \pm 12.41$ & & \\
\hline & Nursing institute & $70.00 \pm 17.72$ & & \\
\hline & Nursing college & $77.78+16.27$ & & \\
\hline
\end{tabular}

Table (4-15) comparison of nurses practices according to their level of education by using ANOVA -test

\begin{tabular}{|c|c|c|c|c|}
\hline level of education practices & level of education & Mean \pm SD & $\begin{array}{l}\text { F- } \\
\text { calculated }\end{array}$ & $\begin{array}{l}\text { Level o } \\
\text { sig. }\end{array}$ \\
\hline \multirow[t]{4}{*}{ Blood pressure measurement } & $\begin{array}{l}\text { Non degree trainin } \\
\text { course }\end{array}$ & $46.67 \pm 18.86$ & 6.49 & \multirow[t]{4}{*}{$<0.05$} \\
\hline & School of nursing & $64.44 \pm 11.09$ & \multirow{7}{*}{1.65} & \\
\hline & Nursing institute & $75.33 \pm 10.45$ & & \\
\hline & Nursing college & $70.73 \pm 10.60$ & & \\
\hline \multirow[t]{4}{*}{ Oxygen administration } & $\begin{array}{l}\text { Non degree trainin } \\
\text { course }\end{array}$ & $66.67 \pm 15.71$ & & \multirow[t]{4}{*}{$\begin{array}{l}>0.05 \\
(\mathrm{NS})\end{array}$} \\
\hline & School of nursing & $71.19 \pm 10.79$ & & \\
\hline & Nursing institute & $77.78 \pm 9.07$ & & \\
\hline & Nursing college & $66.67 \pm 15.71$ & & \\
\hline \multirow{4}{*}{ Cannula insertion } & $\begin{array}{l}\text { Non degree trainin } \\
\text { course }\end{array}$ & $82.14 \pm 13.68$ & \multirow[t]{4}{*}{1.00} & \multirow[t]{4}{*}{$\begin{array}{l}>0.05 \\
(\mathrm{NS})\end{array}$} \\
\hline & School of nursing & $81.48 \pm 10.34$ & & \\
\hline & Nursing institute & $85.71 \pm 6.73$ & & \\
\hline & Nursing college & $77.78 \pm 10.38$ & & \\
\hline
\end{tabular}

Table (4-16) comparison of nurses practices according to their number of employers by using ANOVA -test

\begin{tabular}{l|lrll}
\hline No. of employers / practices & $\begin{array}{l}\text { No. } \\
\text { persons }\end{array}$ & o Mean \pm SD & $\begin{array}{l}\text { F- } \\
\text { calculated }\end{array}$ & Level of sig. \\
\hline & 3 & $71.11 \pm 11.49$ & 1.10 & \\
Blood pressure measurement & 6 & $63.33 \pm 14.69$ & & $>0.05$ (NS)
\end{tabular}




\section{Mosul Journal of Nursing, Vol. 6, No. 2, 2018 ( 100-111 )}

Oxygen administration

Cannula insertion
7

$3 \quad 75.00 \pm 6.91$

$6 \quad 72.22 \pm 11.88$

$7 \quad 69.63 \pm 13.67$

$3 \quad 80.95 \pm 11.12$

$6 \quad 83.93 \pm 9.16$

$7 \quad 81.43 \pm 10.03$
$0.86<0.05$

$0.24<0.05$

Table (4-17) comparison of nurses practices according to their time of work by using ANOVA -test

\begin{tabular}{l|cccc}
\hline Time of work / practices & No. & o & Mean \pm SD & F-calculatec Level of sig. \\
& persons & & & \\
\hline \multirow{3}{*}{ Blood pressure measurement } & day & $64.86 \pm 13.85$ & 1.00 & \\
& evening & $68.33 \pm 11.13$ & & $>0.05$ (NS) \\
Oxygen administration & night & $73.33 \pm 12.47$ & & \\
& day & $70.27 \pm 13.37$ & 0.97 & $>0.05$ (NS) \\
& evening & $72.22 \pm 5.94$ & & \\
night & $77.78 \pm 7.86$ & & \\
Cannula insertion & day & $82.24 \pm 9.76$ & 1.44 & $>0.05(\mathrm{NS})$ \\
& evening & $76.79 \pm 13.09$ & & \\
& night & $85.71 \pm 0.00$ & &
\end{tabular}

\section{Discussion :}

*Socio - demographic characteristics of the sample :

The nurse plays an effective and great role in different types of care for the patients . there is no doubt that the nurse who works in the CCU and ED must be intelligent enough and qualified for these tasks in addition to his general duties, therefore, he must have a good scientific back ground about the profession and details related to coronary artery disease and critical care in order to identify the problems from which the patients and which make the proper decision for intervention. it is clear that the mean age of nurses was (33.86), regarding the gender reveals that distribution of nursing staff according to sex (88\%) male, $12 \%$ female, regarding the place of work, the nurses working in $\mathrm{CCU}$ constituted $(52 \%)$ while in emergency units $(48 \%)$. the problems in emergency department overcrowding. Regarding the duration of experience, less than 5 years constituted $70 \%$, as they are recently employed. regarding the training sessions no $=36 \%$, yes $=64 \%$, these results reveals most of nurses have trained and have advancement in $\mathrm{CCU}$ and $\mathrm{ED}$, regarding the level of ducation, non degree training course $8 \%$, school of nursing $54 \%$, nursing institute $20 \%$. Nursing college $18 \%$ the duration of employment in nursing practice and the level of education affect the performance of nurse, the staff should have sufficient information, high training . in dealing with such disease,

\section{*knowledge and practices of nurses about cardiogenic shock}

Table (2,3) show significant difference regarding the knowledge and practices of nurse about cardiogenic shock, with theoretical mean by using $\mathrm{t}$ - test, in area of information about car of cardiogenic shock, information about medication, general nursing information and the practices area of blood pressure measurement, oxygen administration and cannula insertion. These results indicate the nurses ability to perform efficiently, safely, and multi- disciplinary collaboration in the care of patients during the work in the critical care units(hennery, 1991)

Table 4 show significant difference during care cardiogenic shock and other cases of diseases in the blood pressure measurement . this result reveals nurses who have high level of accountability for decisions that affect clinical practice, and appropriate 


\section{Mosul Journal of Nursing, Vol. 6, No. 2, 2018 ( 100-111 )}

treatments as demanded by the physician in an accurate and timely manner(hennery, 1991).

Variables of nurse with knowledge and practices:

1- Age of nurse: one of the important variables that directly effect patient care in CCU and ED is the age of nurse; using ANOVA test it appear that there no significant difference in knowledge with cardiogenic shock . information about cardiogenic shock treatment, information about medications and general nursing information. Despite the fact the older nurse has more experience and the nurses a ware of different practical measures in minimizing the problems that might happen and provide the best nursing care for patients stricken by cardiogenic shock, in addition, there were no significant difference of practices regarding blood pressure measurement and oxygen administration, these results may be explained by the fact that the nurses were exposed to limited practical tasks, while for cannula insertion, there was significant difference, as this task is gained by prolonged experience.

2-The gender: table 7, 8 show that there are no significant difference in knowledge and practices with regard to sex of nurse, the fact that information included in the study sample, sufficient, and appropriate to the cognition abilities of both the sexes.

3-place of work: regarding the impact of place of work on the knowledge and practices of nurses in table $(9,10)$ which reveled no statistical difference in knowledge and no statistical difference in practices, due to the number of nurses in CCU more than nurse in ED. But is significant regarding general nursing information . one of the most important nursing responsibilities in The CCU is monitoring and ensuring the quality of care, with in this responsibilities are all activities associated with professional practice such as self monitoring the effect of medical intervention and supervising . the emergency nurse practitioners expanding scope of practice has moved beyond managing the care of patients with minor injuries, to include those with minor illnesses and indeed beyond that to the management and care of those with increasingly complex, acute and chronic condition (brook, 2004).

4-duration of employment :the investigator divided the sample into three group according to the yearsof employment in the CCUand ED. Table 11, 12 shows significant statistical difference between the 3 groups and practices . this results is due to he fact that nurses through their presence in the hospital and experience which are gained by continuous care for patients, as well as joining some of the training and development courses which lead to development of their skills and their scientific and practical experiences, maintain high level from expertise in nursing a cross CCU and ED.

5-training sessions: table 13, 14 show no significant difference between knowledge , practical and training sessions . the training sessions is important in developing he work of nurse, the nursing staff should have an advanced level of skills to provide safe and excellent patient care. Where explained the studies that the tension and discomfit in nurses during the work resulted from decrease in the traning

6-level of education: in order to know standards of nurses education, the researcher divided the sample into four groups according to nurses graduation. The purpose of level of education is to provide nurses with he knowledge and skills necessary to diagnose and mange medical emergencies, to provide nurses with an understanding of the social impact of critical illness and the contribution of patient behavior to these problems and to teach the nurse basic diagnostic and interventional procedures (collines.2000). several studies show that the nurses with more education deliver more cost effective care and it is not always easy for nurse with higher education to remain in clinical and public health nurses and there are often no senior position available with in the system, so that nurses with higher education do not reach a position where 
they can implant change and are not reward for there additional education by appropriate remuneration or promotion (kockrow.1999).

Conclusion and Recommendations:

There was significant difference between the knowledge and practices of nurses with regard to theoretical mean. The level of education had a positive impact on the knowledge and practices of nurses who are involved of the study . the gender and the place of work have no significant on knowledge and practices

\section{Recommendation :}

The nurse in these units should have sufficient practices and updated knowledge about critical care especially about cardiogenic shock, nursing stnaff working in the care unit $\mathrm{CCU}$ and ED should be assessing after tanning, patients with ischemic heart disease must be educated about their illness to avoid complication of heart attacks, the nursing staff can do this, it is better to provide each patient, larger study is needed to elaborate he effects of different variables on the knowledge and practices of nurses.

\section{References}

Acley ,BJ, and Ladwig G.B , nursing diagnosis hand book, aguid to planning care. $6^{\text {th }}$ edition, ST, Louis mosby, 2004 Admot R, under standing shock , British jornal of nursing, vol,1,(3) 1992

Ardagh ,M, the circulation and shock, emergency medicne, Christ charge school of medicine, 2000

Beuninger,C,C, Dewitt , P, and Ginnoa $T$, responding to crisis, copy right, Pennsylvania, springhouse, 1993 Brook, S, and Crouch $\mathrm{R}$, doctors and nurse in emergency care; where are the boundaries now, Trauma, 20004
Brunner , and Charlote, Little Rock critical care course, hemodynamic monitoring, lectures notes, 1998

Bunker , C, and Knowalski M.T , Text book of basic nursing, $8^{\text {th }}$ edition, Philadelphia , Lippincott, 2003

Chavez J.A, and Caputo, J, dealing with death and dying, professional learning series , Pennsylvania , 2000

Clark ,T, and Carmen ,G, emergency medicine a comprehensive review, $3^{\text {rd }}$ edition, new York, ravan press 1993

Dejong ,M.J, cardiogenic shock : changes in vital signs may signal impending circulatory collapse, American journal of nursing, vol 97, no(6) , june, 1997

Dens ,J, and Vann DE, W,F, cardiogenic shock, a call for aggressiveness, the European society odf cardiology, vol 21, issue 23, December 2000

Elyes , M,O, comprehensive review of practical nursing for nclex -pn, 13 edition, st Louis, mosoby , 2001

Gawlinskim A. , and Debora, H, Acute care nurse practitioners, clinical curriculum, and cerification review, copyright, Philadelphia W,B, 1999

Goldman , L, and Ausiello,D, Text book of medicine, $2^{\text {nd }}$ edition, W.B, Philadelphia, 2004

Guzzetta ,C, and Montgomer , B, cardiovascular nursing , holistic practice, copy right, ST , Louis, Mosby, 1999

Harkreader ,H, fundamental of nursing, copy right, Philadelphia, W,b, saunders, 2000

Holmes DR, Jr , lethal complicatin of acute myocardial infarction, reviews in cardiovascular medicine, vol 4 , no 3 , 2003

Smltser ,S.C, and Bare, B,G, Text book of medical - surgical nursing , $\mathbf{1 0}^{\text {th }}$ edition , Philadelphia , Lippincott Williams ,2004 